\title{
The delta-shaped anastomosis in laparoscopic distal gastrectomy: analysis of the initial 100 consecutive procedures of intracorporeal gastroduodenostomy
}

\author{
Seiichiro Kanaya • Yuichiro Kawamura • \\ Hironori Kawada $\cdot$ Hironori Iwasaki • \\ Takashi Gomi $\cdot$ Seiji Satoh $\cdot$ Ichiro Uyama
}

Received: 3 February 2011/Accepted: 29 March 2011/Published online: 15 May 2011

(c) The International Gastric Cancer Association and The Japanese Gastric Cancer Association 2011

\begin{abstract}
Background We developed a new method of intracorporeal gastroduodenostomy, the delta-shaped anastomosis, in which only endoscopic linear staplers are used. In this report, we present the short- and long-term outcomes of our initial experience with this procedure.

Methods We retrospectively analyzed 100 consecutive gastric cancer patients who underwent the delta-shaped anastomosis procedure from May 2001 to November 2006. All of them underwent a laparoscopic distal gastrectomy with regional lymph node dissection. Quality of life was assessed with a questionnaire 6 months or more postoperatively, and the gastric remnant was evaluated by endoscopy one year following the surgery.

Results Eight surgeons successfully performed the deltashaped anastomosis without any conversion to laparotomy. The learning curve for all surgeons was steep and the mean time for the anastomosis was $13 \mathrm{~min}$. Only one patient
\end{abstract}

S. Kanaya $(\bowtie) \cdot$ Y. Kawamura · S. Satoh · I. Uyama

Department of Surgery, Fujita Health University

School of Medicine, 1-98 Dengakugakubo,

Kutsukake-cho, Toyoake 470-1192, Japan

e-mail: kanaya@home.email.ne.jp

S. Kanaya $\cdot$ Y. Kawamura - H. Kawada $\cdot$ H. Iwasaki - T. Gomi Department of Surgery, Himeji Medical Center,

68 Hon-machi, Himeji 670-8520, Japan

H. Kawada

Department of Surgery, Kyoto University Graduate School of

Medicine, 54 Shogoin Kawahara-cho, Sakyo-ku,

Kyoto 606-8507, Japan

H. Iwasaki

Department of Cancer Therapy and Research,

Kyushu University Graduate School of Medical Sciences,

3-1-1 Maidashi, Higashi-ku, Fukuoka 812-8582, Japan developed an anastomotic leak, and the leak was minor. Sixty-five patients tolerated a $1500 \mathrm{kcal} / \mathrm{day}$ soft diet at the time of discharge. The mean follow-up period was 54.9 months. Only one patient reported symptoms indicative of dumping. Two patients were diagnosed with reflux esophagitis, and approximately $70 \%$ had evidence of bile reflux, but severe gastritis of the remnant stomach on endoscopy was uncommon.

Conclusions The wide lumen of the delta-shaped anastomosis led to early, adequate postoperative oral intake without a significant incidence of dumping syndrome. The delta-shaped anastomosis is safe and simple and provides satisfactory postoperative results.

Keywords Laparoscopic distal gastrectomy .

Delta-shaped anastomosis · Intracorporeal

gastroduodenostomy · Gastric cancer

\section{Introduction}

Surgery is the mainstay of gastric cancer treatment. A critical objective for gastric surgeons in both the curative and palliative settings is the development of less invasive procedures that result in an improved quality of life. Hence, laparoscopy-assisted gastrectomy is becoming increasingly prevalent. While it is considerably less invasive than the traditional laparotomy approach, the laparoscopy-assisted procedure still requires an additional incision, which is larger than a trocar site, through which to perform the anastomosis. Theoretically, however, this incision can be omitted if an intracorporeal anastomosis is performed, which, presumably, would produce less postoperative morbidity. Historically, intracorporeal anastomosis has been considered a technically difficult and unsecure 
procedure. However, a purely laparoscopic procedure can provide a safe anastomosis independent of the patient's habitus. The establishment of a novel, secure, intracorporeal anastomosis, therefore, is one priority in gastric surgery. We have developed a new, simple, and secure method of intracorporeal gastroduodenostomy, using only endoscopic linear staplers, which we have named the deltashaped anastomosis [1]. Because of its advantages, this procedure is gaining widespread acceptance among surgeons in Japan and Korea, where it is becoming a standard reconstruction procedure after laparoscopic distal gastrectomy [2-5]. In this report, we present the short- and longterm outcomes of our initial series of 100 consecutive procedures.

\section{Patients and methods}

\section{Patients}

One hundred consecutive gastric cancer patients who underwent the delta-shaped anastomosis procedure from May 2001 to November 2006 at Himeji Medical Center, Japan, were enrolled in this study.

All classifications, such as staging and lymph node numbering and grouping, were defined according to the Japanese classification of gastric carcinoma [6].

\section{Delta-shaped anastomosis}

Under general anesthesia, the patient was placed in the reverse Trendelenburg position with the legs apart. Five trocars were placed, as shown in Fig. 1. A laparoscopic distal gastrectomy was then performed with a $\mathrm{CO}_{2}$ pneumoperitoneum. Sufficient lymphadenectomy is performed and the stomach is transected. The resected specimen is removed through the extended umbilical incision, using a large plastic bag. An approximately 4-cm longitudinal incision is enough to remove the specimen, even for advanced gastric cancer. Hence, no additional incision was necessary. The extended umbilical incision normally shrinks well within a few months. Following the gastric resection, the delta-shaped anastomosis was completed as described previously [1]. Our first choice of reconstructive procedure is the Billroth-I with the delta-shaped anastomosis. In the case of a small stomach remnant or short first portion of the duodenum, in which the anastomosis would be under tension, or when gastro-esophageal reflux from a hiatal hernia is anticipated, a Roux-en-Y or Billroth-II reconstruction is selected.

The procedure for the delta-shaped anastomosis consists of the following steps. The duodenal bulb is first transected, using an endoscopic linear stapler from the posterior to the

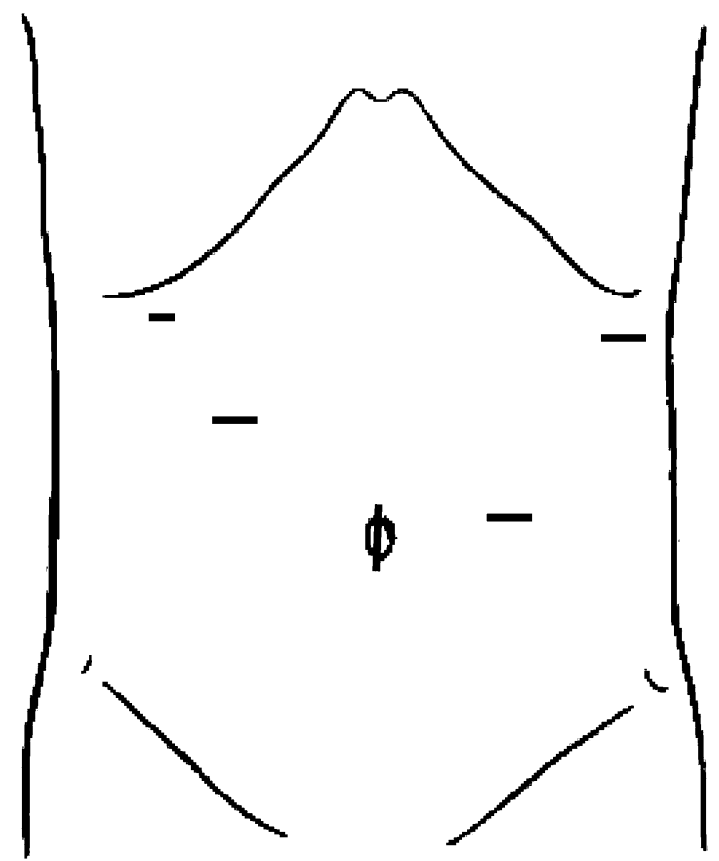

Fig. 1 Trocar placement. The endoscopic linear stapler is inserted through the left lower trocar

anterior wall, at a $90^{\circ}$ angle from the usual line. This preserves the blood supply to the anastomotic line. The stomach is then divided in the usual fashion, from the greater to the lesser curvature. Small incisions are then created along the edge of the stomach and the duodenum (Fig. 2a). The posterior walls of both the stomach and the duodenum are approximated and joined with a $45-\mathrm{mm}$ linear stapler (Fig. 2b). The staple line is then inspected for any defects and hemostasis is verified (Fig. 2c); the enterotomy is then closed with two applications of the linear stapler (Fig. 2d). Kocher's maneuver is never performed as it can lead to rapid gastric transit and dumping syndrome.

\section{Postoperative follow-up}

The nasogastric tube is removed immediately following the procedure and all stable patients, without significant medical co-morbidities, are transferred to the general ward for recovery. When this procedure was first implemented, oral intake was not initiated until the patient had a bowel movement. Since the introduction of a perioperative surgical care pathway for this surgery, from July 2006, patients have been started on clear liquids on postoperative day 1 , and advanced to a soft diet on postoperative day 3 . Once discharged, patients are followed on an outpatient basis every 6 months.

Quality of life was assessed 6 months or more postoperatively, with a questionnaire focusing on dumping syndrome symptoms and microgastria. Analysis was based on 
Fig. 2 Schematic outline of the delta-shaped anastomosis procedure. a Small incisions are placed in the remnant stomach and the duodenum. $\mathbf{b}$ The posterior walls are approximated and the stapler is fired. c Completed V-shaped anastomosis. d Closure of the common stab incision


Table 1 Relative weight of each sign or symptom of dumping syndrome [7]

\begin{tabular}{lc}
\hline Pre-shock, shock & +5 \\
"Almost fainting", syncope, unconsciousness & +4 \\
Desire to lie or sit down & +4 \\
Breathlessness, dyspnea & +3 \\
Weakness, exhaustion & +3 \\
Sleepiness, drowsiness, yawning, apathy, falling asleep & +3 \\
Palpitation & +3 \\
Restlessness & +2 \\
Dizziness & +2 \\
Headache & +1 \\
Feeling of warmth, sweating, pallor, clammy skin & +1 \\
Nausea & +1 \\
Fullness in the abdomen, meteorism & +1 \\
Borborygmus & +1 \\
Eructation & -1 \\
Vomiting & -4 \\
\hline
\end{tabular}

Sigstad's score (Table 1) [7]. The clinical diagnostic indices of dumping syndrome were then calculated. In addition, the gastric remnant was evaluated endoscopically 1 year postoperatively using the RGB (Residue, Gastritis, Bile) classification (Table 2) [8].

\section{Results}

All 100 patients in this study underwent a laparoscopic distal gastrectomy and a regional lymph node dissection. There were 66 men and 34 women. The mean age was 65.5 years. The final stage was IA in 80 patients, IB in 10 , II in 4 , and IIIA in 6 . The lymph node dissection performed was $\mathrm{D} 1+\beta$ in 72 and $\mathrm{D} 2$ in 28 patients. Ninety-four patients underwent a curative potential A resection and six a B resection. The laparoscopic lymphadenectomy was nerve-sparing, as previously described [9]. Both the hepatic and celiac branches of the vagus nerve were preserved in most of the patients with early gastric cancer. Patient characteristics are shown in Table 3.

All operations were completed laparoscopically. Eight surgeons performed the delta-shaped anastomosis procedure. The mean time required for the anastomosis was 13 min (Table 4). Learning curves are shown in Fig. 3. All surgeons adapted quickly to the procedure. There was no intraoperative mortality. Only one case of minor leakage occurred as an anastomosis-related complication, and the patient recovered well with conservative management. Patients were able to tolerate clear liquids at a mean of 2.2 days after the procedure and a soft diet was initiated at a mean of 3.9 days after the procedure (Table 5). The mean postoperative length of hospital stay was 16.7 days; this 
Table 2 RGB (Residue, Gastritis, Bile) classification [8]

\begin{tabular}{|c|c|}
\hline \multicolumn{2}{|c|}{ Residual food } \\
\hline Grade 0 & No residual food \\
\hline Grade 1 & A small amount of residual food \\
\hline Grade 2 & $\begin{array}{l}\text { A moderate amount of residual food, } \\
\text { but possible to observe entire surface } \\
\text { of the remnant stomach with body rolling }\end{array}$ \\
\hline Grade 3 & $\begin{array}{l}\text { A moderate amount of residual food, } \\
\text { which hinders observation of entire } \\
\text { surface even with body rolling }\end{array}$ \\
\hline Grade 4 & $\begin{array}{l}\text { A great amount of residual food, } \\
\text { for which endoscopic observation } \\
\text { is impossible }\end{array}$ \\
\hline \multicolumn{2}{|c|}{ Gastritis (degree) } \\
\hline Grade 0 & Normal mucosa \\
\hline Grade 1 & Mild redness \\
\hline Grade 2 & $\begin{array}{l}\text { Intermediate grade between grade } 1 \\
\text { and grade } 3\end{array}$ \\
\hline Grade 3 & Severe redness \\
\hline Grade 4 & Apparent erosion \\
\hline \multicolumn{2}{|l|}{ Bile reflex } \\
\hline Grade 0 & Absent \\
\hline Grade 1 & Present \\
\hline
\end{tabular}

Table 3 Patient characteristics and operative records

\begin{tabular}{ll}
\hline No. of patients & 100 \\
Age (years, mean $\pm \mathrm{SD})$ & $65.5 \pm 9.3$ \\
Sex (male: female) & $66: 34$ \\
Stage (IA:IB:II:IIIA) & \\
${\text { Ln dissection }(D 1+\beta: D 2)^{\mathrm{a}}}^{\mathrm{a}}+\mathrm{80:10:4:6}$ \\
${\text { Curative potential }(\mathrm{A}: \mathrm{B}: \mathrm{C})^{\mathrm{a}}}^{\text {Preservation of vagus nerve }(\mathrm{H}+\mathrm{C}: \mathrm{H}: \text { None) }}$ & 72.28 \\
\hline
\end{tabular}

$H$ hepatic branch, $C$ celiac branch of vagus nerve, $L n$ lymph node

${ }^{a}$ Classified according to the Japanese classification of gastric carcinoma $[6]$

Table 4 Operative data

\begin{tabular}{lc}
\hline Operative time $(\mathrm{min}$, mean $\pm \mathrm{SD})$ & $239.2 \pm 53.2$ \\
Anastomotic time $(\mathrm{min}$, mean $\pm \mathrm{SD})$ & $13.0 \pm 3.9$ \\
Blood loss $(\mathrm{ml}$, mean $\pm \mathrm{SD})$ & $92.6 \pm 89.7$ \\
Conversion to other procedures & None \\
\hline
\end{tabular}

duration was reduced to around 10 days after the perioperative surgical care pathway was incorporated in the postoperative management. Figure 4 shows the volume of oral intake tolerated at the end of the hospital stay. Most patients tolerated a solid diet, with two-thirds of the patients consuming $100 \%$ of the $1500 \mathrm{kcal} / \mathrm{day}$ soft diet at discharge.
With a mean follow-up of 54.9 months (range 28-94 months), there were three cancer recurrences, two paraaortic lymph node recurrences, and one liver metastasis. Meanwhile, one patient died of a non-cancer related cause, and two died from non-gastric cancers. In addition to these, 13 patients were lost to follow-up, yielding a final response rate for the questionnaire of $78 \%$. Subsequently, only one patient was diagnosed with dumping syndrome, based on a diagnostic index equal to or greater than +7 . Seventy-four patients (94.9\%) were diagnosed as not having dumping syndrome, based on an index equal to or less than +4 (Fig. 5). Table 6 shows the frequency distribution of postprandial symptoms. While regurgitation and eructation were rather frequent, five patients reported heartburn and only one patient required medical management of reflux.

Figure 6 shows a graphical representation of the results of the endoscopic examination, which was performed in 83 patients. Two patients were diagnosed with reflux esophagitis. While $73.5 \%$ of the patients demonstrated bilious reflux into the remnant stomach, only 15 patients (23.8\%) had second degree or worse remnant gastritis.

\section{Discussion}

In this study, we have demonstrated that the delta-shaped anastomosis is a simple, easy and safe method of intracorporeal gastroduodenostomy. Eight surgeons performed all procedures as planned, without any conversion to other procedures or laparotomy. The mean time for the anastomosis was only $13 \mathrm{~min}$ and the learning curves were remarkably steep.

The Billroth-I procedure is generally believed to be the easiest reconstruction, as it only requires one anastomosis; however, it is also believed that complications after Billroth-I reconstruction occur more frequently than after other reconstructions and are likely to become serious [10]. In the present study, however, the rate of anastomosis-related complications was lower than what has previously been reported [2, 10], with only one patient experiencing a minor leakage, which responded to conservative management. Our experience with these 100 cases strongly suggests that the delta-shaped anastomosis is a simple and safe form of intracorporeal gastroduodenostomy.

To confirm that the oral intake in the early postoperative period was adequate, food and liquid intake was measured at the end of the hospitalization. Two-thirds of the patients ate $100 \%$ of the $1500 \mathrm{kcal} / \mathrm{day}$ soft diet. It is likely that the wide lumen of the delta-shaped anastomosis promoted oral intake in the early postoperative period. While a larger lumen facilitates the passage of solid food, it does increase the risk of dumping syndrome, particularly when the lumen 
Fig. 3 Times for anastomosis. Eight surgeons performed the delta-shaped anastomosis procedure. The time plateaued at around $10 \mathrm{~min}$ within a few experiences



Dr.A Dr.B $=$ Dr.C Dr.D Dr.E Dr.F Dr.G Dr.H
Table 5 Postoperative outcome

\begin{tabular}{ll}
\hline Clear liquids initiated (days, mean $\pm \mathrm{SD})$ & $2.2 \pm 1.1$ \\
Soft diet initiated (days, mean $\pm \mathrm{SD})$ & $3.9 \pm 1.0$ \\
Postoperative length of hospital stay (days, mean \pm SD) & $16.7 \pm 13.8$ \\
(since July 2006 & $10.1 \pm 4.0)$ \\
Complications & Minor leakage 1 \\
\hline
\end{tabular}

a Improved by fasting

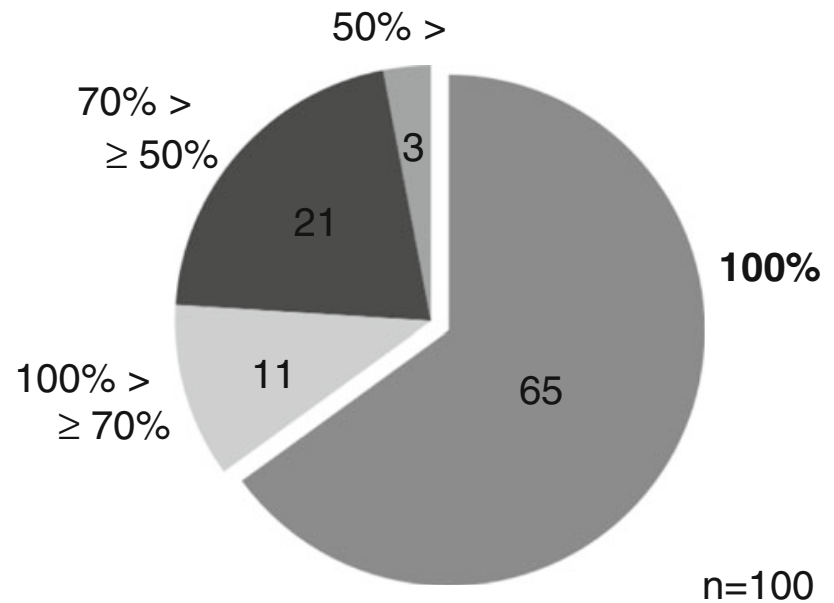

Fig. 4 Volume of oral intake at the end of hospital stay. Two-thirds of the patients ate $100 \%$ of the hospital diet, a $1500 \mathrm{kcal} / \mathrm{day}$ soft diet

is overly large. Dumping syndrome symptoms were assessed in the postoperative period with a standard symptom questionnaire. As both early and late dumping syndrome result from rapid gastric emptying, we did not distinguish between the two subtypes when analyzing Sigstad's scores [7]. Based on the analysis of the questionnaire responses, only one patient was diagnosed with dumping syndrome. This may be explained by the following postoperative fluoroscopic and endoscopic findings. In the early postoperative period, contrast passes readily, which is consistent with the rapid resumption of adequate



Fig. 5 Clinical diagnosis index of the dumping syndrome from analysis of the questionnaires that were sent to the patients more than 6 months after the operation. Border borderline dumping syndrome

oral intake following surgery. Fluoroscopy a few months later, however, shows some retention of contrast material around the anastomotic site, with delayed passage. Endoscopy performed around the same time did not demonstrate any retained food and the lumen appeared to be of adequate diameter. The brief stasis evident on fluoroscopy may have been the result of duodenal twisting at the site of the delta-shaped anastomosis. This stasis may be sufficient to prevent dumping syndrome in most patients.

While only one patient was diagnosed with dumping syndrome, fifteen $(19.2 \%)$ were diagnosed with gastroesophageal reflux, based on a Sigstad's score of less than 0 , and most patients reported "regurgitation" and "eructation". Patients infrequently experienced heartburn (6.7\%) and only two patients had endoscopic evidence of reflux esophagitis. On the other hand, bile reflux into the remnant stomach was observed endoscopically in $73.5 \%$ of our patients, a rate higher than what has previously been 
Table 6 Postprandial symptoms

\begin{tabular}{lr}
\hline Palpitations & 4 \\
Fatigue & 4 \\
Dizziness & 3 \\
Syncope & 1 \\
Sweating & 4 \\
Headache & 1 \\
Paleness & 1 \\
Abdominal pain & 6 \\
Diarrhea & 19 \\
Nausea & 7 \\
Vomiting & 6 \\
Borborygmus & 8 \\
Meteorism & 2 \\
Tremor & 1 \\
Almost fainting & 2 \\
Cold sweating & 2 \\
Sleepiness & 5 \\
Hunger & 1 \\
Regurgitation & 8 \\
Heartburn & 24 \\
Eructation & 5 \\
Hiccups & 20 \\
Abdominal fullness & 1 \\
\hline
\end{tabular}
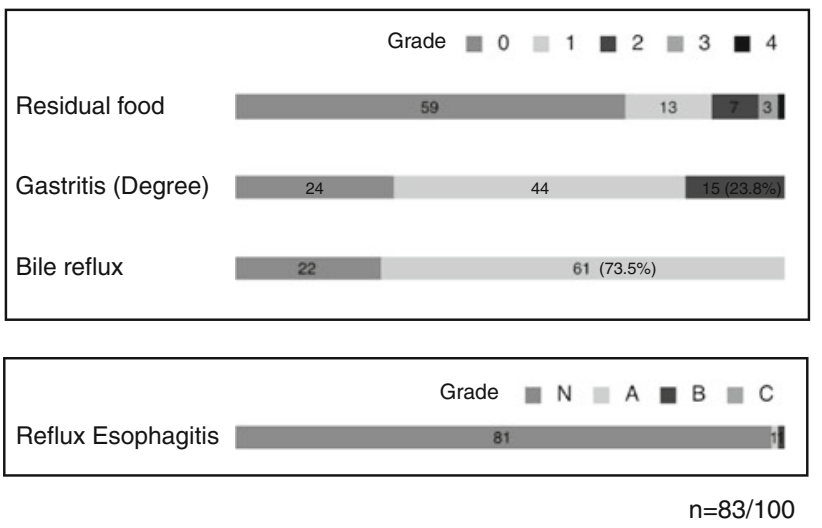

Fig. 6 Results of the RGB (Residue, Gastritis, Bile) classification and presence of reflux esophagitis on endoscopy 1 year postoperatively

reported $[8,10,11]$. However, the incidence of remnant gastritis was low and severe symptoms were rarely observed. Thus, we concluded that while bile reflux was prevalent, it did not appear to be a clinically critical problem in our series.

A limitation of a completely laparoscopic technique is the difficulty of locating the tumor intraoperatively. Hyung et al. [12] reported that the resection line could be defined based on a combination of preoperative endoscopic clipping and intraoperative laparoscopic ultrasonography. Tanimura et al. [13] employed preoperative endoscopic inking. We used intraoperative endoscopy to identify the tumor and generally performed a total gastrectomy in any patients with tumor invasion in the upper third of the stomach. Another criticism of the laparoscopic distal gastrectomy with the delta-shaped anastomosis is its higher cost, primarily because it requires up to seven linear stapler cartridges to complete. Although this is an economically perplexing issue, this procedure has various advantages compared with other reconstructions. The smaller wound means a better cosmetic result and may lead to less invasiveness. In addition, purely laparoscopic surgery can provide a safe anasomosis independently of the patient's habitus and brings about good postoperative respiratory function [14]. The delta-shaped anastomosis is safe in terms of minimal postoperative morbidity, and it is easy to perform, at least with the support of a well-experienced surgeon.

In conclusion, while an additional prospective clinical study should be planned to confirm the safety and efficacy of this procedure, the present review does support the idea that the delta-shaped anastomosis is simple and safe, and produces satisfactory short- and long-term results.

\section{References}

1. Kanaya S, Gomi T, Momoi H, Tamaki N, Isobe H, Katayama T, et al. Delta-shaped anastomosis in totally laparoscopic Billroth I gastrectomy: new technique of intraabdominal gastroduodenostomy. J Am Coll Surg. 2002;195:284-7.

2. Kim JJ, Song KY, Chin HM, Kim W, Jeon HM, Park CH, et al. Totally laparoscopic gastrectomy with various types of intracorporeal anastomosis using laparoscopic linear staplers: preliminary experience. Surg Endosc. 2008;22:436-42.

3. Ikeda O, Sakaguchi Y, Aoki Y, Harimoto N, Taomoto J, Masuda $\mathrm{T}$, et al. Advantages of totally laparoscopic distal gastrectomy over laparoscopically assisted distal gastrectomy for gastric cancer. Surg Endosc. 2009;23:2374-9.

4. Kim MG, Kawada H, Kim BS, Kim TH, Kim KC, Yook JH, et al. A totally laparoscopic distal gastrectomy with gastroduodenostomy (TLDG) for improvement of the early surgical outcomes in high BMI patients. Surg Endosc. 2011;25: 1076-82.

5. Kinoshita T, Shibasaki H, Oshiro T, Ooshiro M, Okazumi S, Katoh R. Comparison of laparoscopy-assisted and total laparoscopic Billroth-I gastrectomy for gastric cancer: a report of shortterm outcomes. Surg Endosc. 2011;25:1395-401.

6. Japanese Gastric Cancer Association. Japanese classification of gastric carcinoma. 2nd English edition. Gastric Cancer. 1998;1: $10-24$.

7. Sigstad H. A clinical diagnostic index in the diagnosis of the dumping syndrome. Changes in plasma volume and blood sugar after a test meal. Acta Med Scand. 1970;188:479-86.

8. Kubo M, Sasako M, Gotoda T, Ono H, Fujishiro M, Saito D, et al. Endoscopic evaluation of the remnant stomach after gastrectomy: proposal for a new classification. Gastric Cancer. 2002;5:83-9. 
9. Uyama I, Sakurai Y, Komori Y, Nakamura Y, Syoji M, Tonomura $S$, et al. Laparoscopic gastrectomy with preservation of the vagus nerve accompanied by lymph node dissection for early gastric carcinoma. J Am Coll Surg. 2005;200:140-5.

10. Kojima K, Yamada H, Inokuchi M, Kawano T, Sugihara K. A comparison of Roux-en-Y and Billroth-I reconstruction after laparoscopy-assisted distal gastrectomy. Ann Surg. 2008;247:962-7.

11. Nunobe S, Okaro A, Sasako M, Saka M, Fukagawa T, Katai H, et al. Billroth 1 versus Roux-en-Y reconstructions: a quality-oflife survey at 5 years. Int J Clin Oncol. 2007;12:433-9.
12. Hyung WJ, Lim JS, Cheong JH, Kim J, Choi SH, Song SY, et al. Intraoperative tumor localization using laparoscopic ultrasonography in laparoscopic-assisted gastrectomy. Surg Endosc. 2005; 19:1353-7.

13. Tanimura S, Higashino M, Fukunaga Y, Osugi H. Laparoscopic distal gastrectomy with regional lymph node dissection for gastric cancer. Surg Endosc. 2003;17:758-62.

14. Tanimura S, Higashino M, Fukunaga Y, Kishida S, Ogata A, Fujiwara Y, et al. Respiratory function after laparoscopic distal gastrectomy-an index of minimally invasive surgery. World $\mathrm{J}$ Surg. 2006;30:1211-5. 